AgNOR counts represent a marker of proliferative activity. ${ }^{4}$ Although there are problems and limitations to mitotic counting, ${ }^{8}$ this method has become widely accepted in the routine assessment of stromal tumours of the gut. $^{12}$ The mitotic count can be performed more quickly and easily than AgNOR staining and uses routinely available haematoxylin and eosin stained sections. There is also a wider variation in the mitotic count than there is for the AgNOR count. Because there is only a small difference in AgNOR count between benign and malignant tumours, it is unlikely that the technique has any practical use for the diagnosis or prognostication in these neoplasms.

We thank Mr B Simms for photographic assistance and Dr EJ Lazda for help with graphics.
1 Evans HL. Smooth muscle tumours of the gastrointestinal tract. A study of 56 cases followed for a minimum of 10 years. Cancer 1985;56:2242-50.

2 Ranchod M, Kempson RL. Smooth muscle tumours of the gastrointestinal tract and retroperitoneum. A pathologic gastrointestinal tract and retroperitoneum. A pathologic
analysis of 100 cases. Cancer 1977;39:255-62.

3 analysis of 100 cases. Cancer 1977;39:255-62.

, Menager $M$, Jeannesson $P$, Himber G, Pigeon $F$ Adnett JJ. Improvement in the staining and in the visualization of the argyrophilic proteins of the nucleolar organizer region at the optical level. Histochem $J$ 1986;18:5-14.

4 Crocker J. Nucleolar organizer regions. In: Underwood JCE, ed. Current topics in pathology. Vol 82: Pathology of the nucleus. Berlin: Springer-Verlag, 1990:91-149.

5 Rowlands DC, Crocker J, Ayres JG. Silver staining of nucleolar organizer region associated proteins using polyethylene glycol as the protective colloidal developer. Histochem J 1990;22:555-9.

6 Crocker J, Boldy DAR, Egan MJ. How should we count AgNORs? Proposals for a standardized approach. J Pathol 1990;158:185-88.

7 Sinn HP, Lehnert T, Kandetzki C, Waldherr R. Nucleolar organizer regions in myogenic stromal tumours of the stomach. Virchows Arch (Pathol Anat) 1989;415:317-21.

8 Quinn CM, Wright NA. The clinical assessment of proliferation and growth in human tumours: evaluation of methods and applications as prognostic variables. J Pathol 1990;160:93-102.

\title{
Salmonella osteomyelitis in aplastic anaemia after antilymphocytic globulin and steroid treatment
}

\author{
S Allard, J O’Driscoll, A Laurie
}

Department of Haematology, St George's Hospital Blackshaw Road, London SW17 0QT $S$ Allard

A Laurie

Department of Microbiology

J O'Driscoll

Correspondence to: Dr S Allard

Accepted for publication 4 July 1991

\begin{abstract}
A 22 year old patient with severe aplastic anaemia responded to antilymphocytic globulin but developed recurrent fever despite treatment with steroids and then antibacterial, antifungal, and antituberculous drugs. There was progression, with severe joint pains and immobility associated with radiological evidence of a symmetrical destructive process. A bone marrow specimen showed no evidence of malignancy, and cultures of blood, urine, and stool were negative but enriched broth cultures of an open biopsy specimen of the humeral head grew Salmonella enteriditis phage type 4. Treatment with ciprofloxacin resulted in considerable symptomatic improvement: a total of 12 months of treatment is planned. Salmonella osteomyelitis, particularly with this unusual pattern of disease, has not previously been described in aplastic anaemia.
\end{abstract}

\section{Case report}

Bone and joint infections due to Salmonella are uncommon, although various predisposing conditions are recognised, particularly sickle cell anaemia. ${ }^{1-3} \mathrm{We}$ describe a patient with aplastic anaemia who responded well to treatment with antilymphocytic globulin (ALG) but who developed severe Salmonella enteriditis (type 4) osteomyelitis.

A 22 year old Greek woman presented with anaemia and thrombocytopenia (haemoglobin $5 \mathrm{~g} / \mathrm{dl}$; platelets $42 \times 10^{9} / 1$; white cell count $3.3 \times 10^{9} / 1$, with $46 \%$ neutrophils, $48 \%$ lymphocytes, and $6 \%$ monocytes), and investigations confirmed idiopathic aplastic anaemia. She received $15 \mathrm{mg} / \mathrm{kg}$ equine ALG (Merrieux) daily for five days infused into the subclavian vein via a line inserted into the antecubital fossa. Five days later a typical syndrome of serum sickness developed with fever, rash, and arthralgia and this responded promptly to hydrocortisone.

High fever with negative blood cultures 


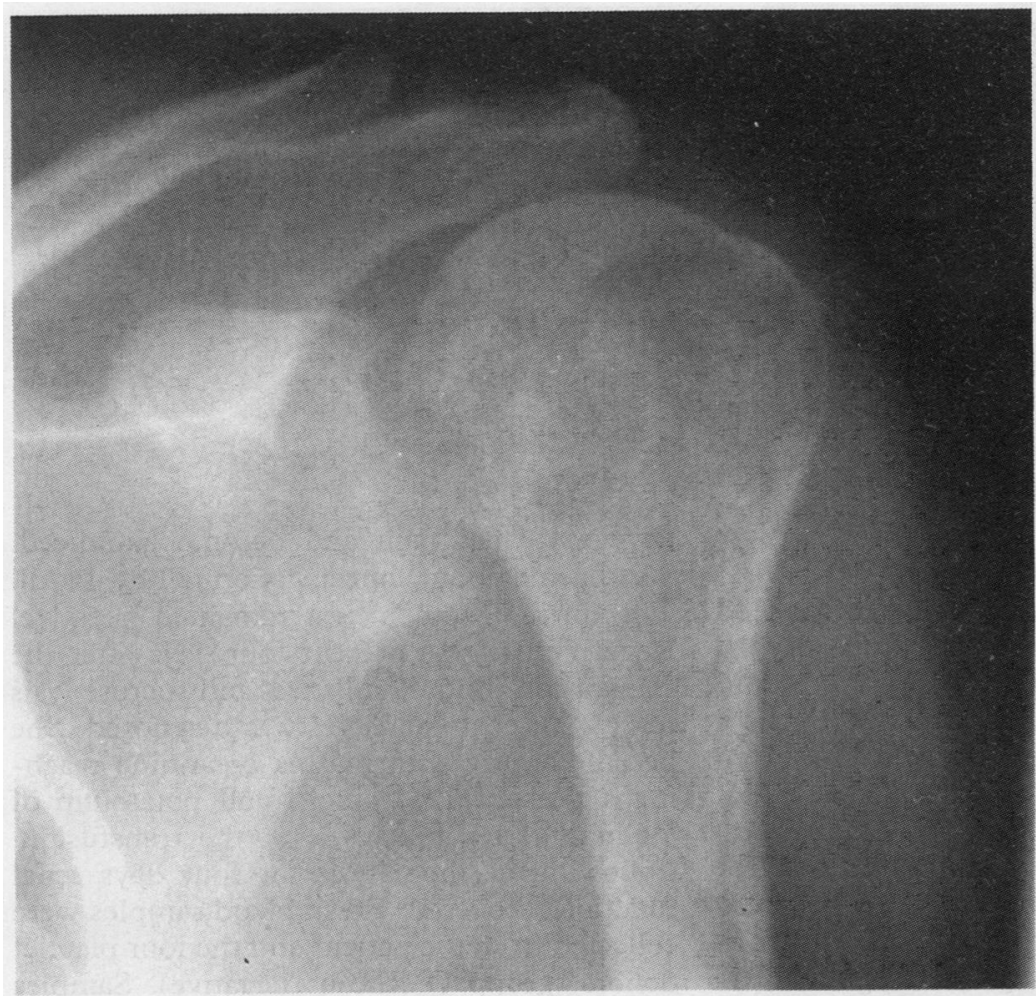

$X$-ray picture of the left shoulder showing destructive bone and joint changes.

recurred, however, despite further treatment with hydrocortisone and subsequent trials of broad spectrum antibiotics, amphotericin B, and finally antituberculous chemotherapy. She then developed pain in the left hip and was given high dose steroids (intravenous methylprednisolone followed by oral prednisolone $60 \mathrm{mg}$ daily). The fever settled but the joint pains progressed in both shoulders with radiological evidence of a destructive process affecting bone and joints.

She was transferred to the United Kingdom where steroids were stopped. Swinging fevers returned; by now she was totally immobilised by pain. No passive movement of the hips and shoulders was possible and coarse crepitus was noted. She was cushingoid with severe muscle wasting aggravated by disuse. By this time she was transfusion independent with a haemoglobin of $10 \cdot 1 \mathrm{~g} / \mathrm{dl}$, platelet count $313 \times 10^{9} / 1$, and a white cell count of $7.0 \times 10^{9} / 1$ with $69 \%$ neutrophils. A bone marrow aspirate (left anterior iliac crest) was normocellular with no evidence of malignancy. The erythrocyte sedimentation rate was $125 \mathrm{~mm} / \mathrm{h}$ and the C-reactive protein was $108 \mathrm{mg} / \mathrm{l}$. Further $x$-ray pictures of the pelvis, hips, and shoulders confirmed a progressive destructive process (figure) with loss of joint spaces and lytic and sclerotic bone changes. All cultures of blood, urine, and stool were negative (including early morning urines for tuberculosis). Brucella serology was negative and Staphylococcus aureus antibodies were $<1$ unit/ml.

An open bone biopsy (left humeral head) was performed in an attempt to make a definitive diagnosis. The bone marrow had an abnormal rubbery texture with a discoloured grey brown appearance. Histological examination of the specimen showed severe chronic inflammation; no organisms or crystals were seen and no neoplastic infiltrate was identified. In view of her deteriorating condition she was given empirical antibiotic treatment with intravenous vancomycin $(1 \mathrm{~g}$ twice a day), intravenous ciprofloxacin (200 $\mathrm{mg}$ twice a day), and oral fusidic acid (500 $\mathrm{mg}$ three times a day).

Enriched broth cultures from the bone biopsy specimen now grew an organism that was identified as Salmonella enteriditis (phage type 4) which was fully sensitive to ciprofloxacin; this was therefore continued as single agent treatment. Over the next three weeks the high fevers settled completely, bone pain at rest resolved, and there was gradual improvement in mobilisation with physiotherapy. She returned to Greece with a plan to continue oral ciprofloxacin $(750 \mathrm{mg}$ twice a day) for a total of 12 months after which joint replacement surgery may be considered.

\section{Discussion}

Salmonella osteomyelitis has been attributed to use of steroids, but such cases generally involve one or two sites only..$^{2-4}$ Cherubin et al described a young woman receiving steroids for systemic lupus erythematosus who developed osteomyelitis due to Salmonella enteriditis after undergoing pin repair of a fracture of the ankle. ${ }^{4}$ In a review of 37 cases of bone infection due to Salmonella, ${ }^{3}$ three patients were receiving immunosuppressive treatment for connective tissue disorders; all three presented with localised osteomyelitis, though there was subsequent involvement of a second site in one case.

Our patient showed an extensive symmetrical osteomyelitis rarely reported except in sickle cell anaemia, ${ }^{12}$ and not previously reported in aplastic anaemia. This unusual clinical picture posed considerable diagnostic difficulties and, we feel, probably reflects impaired immunity due to prolonged steroid treatment aggravated by impaired marrow function. The use of a central venous catheter raises the possibility of a sustained bacteraemia, and it is interesting to speculate whether coincident serum sickness modified the extent of the infection.

1 Adeyokunnu AA, Hendrickse RG. Salmonella osteomyelitis in childhood. Arch Dis Child 1980;55:175-84.

2 Cohen JL, Bartlett JA, Corey GR. Extra-intestinal manifestations of Salmonella infections. Medicine 1987;66: 349-88.

3 Oritz-Neu C, Marr JS, Cherubin CE, Neu HC. Bone and joint infections due to Salmonella. J Infect Dis 1978; 138:820-8.

4 Cherubin CE, Neu HC, Imperator PJ, Harvey RP, Bellen N. Septicaemia with non typhoid Salmonella. Medicine 1974;53:365-76. 